\title{
An OR practitioner's solution approach to the multidimensional knapsack problem
}

\author{
Zachary Kern ${ }^{\mathrm{a}}$, Yun Lu ${ }^{\mathrm{a}}$ and Francis J. Vasko ${ }^{\mathrm{a}^{*}}$
}

${ }^{a}$ Department of Mathematics, Kutztown University, Kutztown, PA 19530, USA

\begin{tabular}{l}
\hline C H R O N I C L E \\
\hline Article history: \\
Received March 52019 \\
Received in Revised Format \\
April 152019 \\
Accepted June 142019 \\
Available online \\
June 142019 \\
\hline Keywords: \\
Mixed-integer programming \\
Payment term \\
Trade credit \\
Logistics \\
Quantity flexible contract \\
Factoring
\end{tabular}

\section{Introduction}

Since the 0-1 Multidimensional Knapsack Problem (MKP) has numerous real-world direct applications or sub-problem applications that need to be solved, it is important for operations research practitioners to use simple, effective and efficient solution approaches for solving such problems. This is true regardless if the OR practitioner is called on to assist with a critical strategic planning issue (e.g., Newhart et al., 1993; Vasko et al., 2005; Vasko \& Stott, 2008) or needs to implement an optimization module in a production system that is executed daily (e.g., Vasko et al., 1989; Vasko et al., 1991; Vasko et al., 1993; Vasko et al., 2005). Since the MKP is NP-hard and most real-world applications are typically large in scale, exact solution approaches are usually not appropriate.

\footnotetext{
* Corresponding author

E-mail: vasko@,kutztown.edu (F. Vasko)

2020 Growing Science Ltd.

doi: $10.5267 /$ j.ijiec.2019.6.004
} 
A comprehensive overview of practical and theoretical results for the MKP can be found in the monograph on knapsack problems by Kellerer et al. (2004). An early review of the MKP was given by Fréville (2004), and a more recent survey of the MKP is given by Laabadi et al. (2018). The 0-1 MKP has been introduced to formulate many practical problems including capital-budgeting problems, transportation problems, allocation of databases and processors in distributed data processing, scheduling of computer programs in multiprogramming environments, investment policies for the tourism sector of developing countries, approval voting, and so on. (Meng \& Pan, 2017; Baghel et al., 2012). Next, we give a mathematical programming formulation for the multidimensional knapsack problem.

The mathematical formulation for the 0-1 Multidimensional Knapsack Problem is:

$$
\max z=\sum_{j=1}^{n} p_{j} x_{j}
$$

subject to

$$
\begin{aligned}
& \sum_{j=1}^{n} a_{i j} x_{j} \leq b_{i} \quad i=1, \cdots, m \\
& x_{j} \in\{0,1\} \quad j=1, \cdots n .
\end{aligned}
$$

Decision variables are binary where $x_{j}=1$ means that item $j$ is packed in the knapsack, and $x_{j}=0$ otherwise. Each item $j$ requires $a_{i j}$ units of resource consumption in the $i$ th knapsack constraint and yields $p_{j}$ units of profit upon inclusion in the knapsack. The goal is to find a subset of items that yields maximum profit without exceeding the resource capacities (the $b_{i} \mathrm{~s}$ ). There are many approximate solution approaches available from the literature. Some recent (since 2011) examples applied to solve the MKP include: harmony search (HS)-based approaches by Kong et al. (2015) and Rezoug and Boughaci (2016), particle swarm optimization (PSO)-based approaches by Labed et al. (2011) and Kang (2012), a shuffled complex evolution algorithm by Baroni and Varejao (2015). fruit fly optimization algorithm by Meng and Pan (2017), or a guided genetic algorithm (GGA) approach by Rezoug et al. (2018). In order to solve the MKP, earlier papers discussed a genetic algorithm by Chu and Beasley (1998) and heuristic approaches by Moraga et al. (2005), Akçay et al. (2007) and Boyer et al. (2009). A classic paper by Freize and Clarke (1984) discussed both probabilistic and worst-case analyses for the MKP.

All the solution approaches mentioned in the previous paragraph require the user to set or fine-tune several parameters. For example, Labed et al. (2011) present a hybrid Particle Swarm Optimization (PSO) and Genetic Algorithm (GA) algorithm which requires the fine-tuning of both PSO parameters such as acceleration coefficients and inertia weight and GA parameters involved in parent selection, crossover operator and mutation operator. Another example of an algorithm requiring considerable finetuning is the fruit fly algorithm of Meng and Pan (2017) which requires the fine-tuning of six parameters. Additionally, harmony search-based algorithms (Kong et al. (2015)) require harmony memory size, harmony memory considering rate, pitch adjustment rate, and pitch adjustment step. In contrast, the twophase Teaching-Learning Based Optimization (TLBO) approach is a population-based metaheuristic developed by Rao et al. (2011) and originally designed to solve continuous nonlinear engineering design optimization problems. This metaheuristic is particularly attractive to practitioners that need to solve real-world problems and do not have time for parameter fine-tuning (and re-fine-tuning if the problem instances change over time). The user simply needs to decide on the population size and when to terminate the process - two things which all population-based metaheuristics must address. We develop an easy-to-implement adaptation of TLBO for the MKP. Our adaptation of TLBO for the MKP uses a simple binarization approach (previously used successfully by Lu and Vasko (2015), Zyma et al. (2015) and Vasko et al. (2016)) to adapt this metaheuristic to solve the binary MKP. Furthermore, unlike many other MKP solution approaches (e.g., Kong et al., (2015)), we do not require the solution of the MKP linear programming relaxation as part of the repair operator. Additionally, assuming that an OR practitioner would have limited time to experiment with population size, we used a population size of 30 
which had worked well when the TLBO was used to solve other binary optimization problems (Lu and Vasko (2015), Zyma et al. (2015) and Vasko et al. (2016)).

In this paper, we used 270 test problems available in Beasley's OR-Library to measure the performance of our TLBO algorithm for the MKP. Furthermore, Rezoug et al. (2018) recently summarized in their Table 10, how 10 solution approaches for the MKP performed on these same 270 test problems. TLBO performed competitively with the 10 solution approaches reported in Rezoug et al. (2018). Specifically, on the problems with the largest number of constraints (usually considered more difficult to solve), TLBO outperformed all but two of the 10 solution procedures, while its results on the best two solution procedures were comparable without the need for time-consuming parameter fine-tuning. The advantage of the TLBO approach is its relative simplicity while generating high-quality solutions effectively. This is especially important for operations research practitioners who need a simple, robust solution approach (that does not need periodic re-fine-tuning) for solving their problems.

In the next section, we will give details of the TLBO metaheuristic. That will be followed by a discussion of the adaptation of this metaheuristic to solve the MKP. Then empirical results will be used to compare our TLBO solutions of the 270 Beasley test problems with the 10 solution approaches that are compared in Rezoug et al.(2018). Finally, a brief summary will be provided.

\section{Teaching-Learning Based Optimization}

The Teaching-learning-based optimization (TLBO) metaheuristic is a two-phase population-based metaheuristic designed to solve continuous nonlinear optimization problems. It was proposed by Rao et al. (2011) as a novel method for solving large constrained mechanical design optimization problems which involve no specific parameters to tune. Since the tuning of parameters in other metaheuristics can often be time consuming and largely experimental, Rao et al. (2011) describe a procedure in which the only parameters that need to be specified are those common to all other metaheuristics--population size and termination criterion. TLBO was inspired by the observation of how learning is done in a typical classroom setting. This is seen as being done in two phases: (1) the teaching phase and (2) the learning phase. The teaching phase attempts to raise the mean quality of a population of students based on the teacher. Factors which impact this process are the quality of the teacher, the capability of the class, and an amount of random or unpredictable behavior. The learning phase mimics how students in a class learn amongst themselves through group discussions, presentations, and so forth. Here, a learner may learn something new if other learners are more knowledgeable than him or her. The first phase of TLBO, the teaching phase, utilizes a global search procedure. The "difference mean" is created by subtracting the quality of the best solution with the current mean solution. The objective here is to improve all solutions by this difference. The operator creating a new solution in the teaching phase is given as the following: $X_{\text {new }}=X_{\text {old }}+r\left(X_{\text {teacher }}-T_{f} \times X_{\text {mean }}\right)$, where $X_{\text {old }}$ is a current solution of a population being modified, $r$ is a random number in the range $[0,1], X_{\text {teacher }}$ is the best solution of a population, $T_{f}=\operatorname{round}(1+\operatorname{rand}(0,1))$ implying that $\mathrm{T}_{\mathrm{f}}$ takes on the values 1 or 2 with equal probability. Also, $X_{\text {mean }}$ is the mean solution of a population (Rao et al, 2011). Here, two variables $r$ and $T_{f}$ could have been used as parameters; however, they are defined as being random numbers and therefore their values are not specified as input parameters. The teaching phase is completed by checking if the new solution is better than the current. The second phase of TLBO adjusts each solution relative to a randomly selected solution (another learner). The operator is given by the following (for a minimization problem):

$$
X_{i, \text { new }}= \begin{cases}X_{i}+r\left(X_{i}-X_{j}\right), & \text { if } f\left(X_{i}\right)<f\left(X_{j}\right) \\ X_{i}+r\left(X_{j}-X_{i}\right), & \text { else }\end{cases}
$$

where, similar to the teaching phase, $r$ is randomly chosen in the range of $[0,1], X_{i}$ is the current solution and $X_{j}$ is a randomly chosen solution where $i \neq j$. For both phases of TLBO, since $\mathrm{X}_{\mathrm{j}}$ is a vector of non- 
negative values, the actual implementation of TLBO requires the use of these update formulas on each component of $\mathrm{X}_{\mathrm{j}}$.

This procedure is given below:

\section{TLBO METAHEURISTIC}

1. Begin

2. $g \leftarrow 0$

3. $X=$ initialize_population $($ pop_size $)$

4. evaluate $(X)$

5. Repeat

6. $X=\operatorname{sort}(X)$ from minimum objective function to maximum

7. For $i=1 \rightarrow$ pop_size do

8. $\quad$ Teacher Phase $\}$

9. $T_{F}=\operatorname{round}(1+\operatorname{rand}(0,1))$

10. $X_{\text {mean }} \leftarrow X_{\text {pop_size / } 2}$

11. $X_{\text {teacher }} \leftarrow X_{1}$

12. $X_{i, \text { new }}=X_{i}+\operatorname{rand}(0,1) \times\left(X_{\text {teacher }}-\left(T_{F} \times X_{\text {mean }}\right)\right)$

13. Evaluate $\left(X_{\text {new }}\right)$

14. If $X_{i}$, ${ }_{\text {new }}$ better than $X_{i}$ then

15. $X_{i} \leftarrow X_{\text {new }}$

16. end if $\{$ End of Teacher Phase $\}$

17. $\quad$ Learner Phase $\}$

18. $\quad i i \leftarrow$ random $($ pop_size $)\{i i \neq i\}$

19. If $X_{i}$ better than $X_{i i}$ then

20. $X_{i, \text { new }}=X_{i}+\operatorname{rand}(0,1) \times\left(X_{i}-X_{i i}\right)$

21. Else

22. $X_{i, \text { new }}=X_{i}+\operatorname{rand}(0,1) \times\left(X_{i i}-X_{i}\right)$

23. $\quad$ End if

24. $\quad$ Evaluate $\left(X_{i, \text { new }}\right)$

25. If $X_{i, \text { new }}$ better than $X_{i}$ then

26. $\quad X_{i} \leftarrow X_{i, \text { new }}$

27. End if $\{$ End of Learner Phase

28. End for

29. $g \leftarrow g+1$

30.Until $(g \neq$ num_gen $)\{$ termination condition $\}$

31. Print_best_result $(X)$

32. End

For more information on TLBO, we suggest reading Rao et al. (2011). 


\section{Details for Implementing TLBO to solve MKPs}

\subsection{Initial Population Generation}

Any population-based metaheuristic requires the generation of an initial population of feasible solutions. The initial population size is set at 30 unique feasible solutions. This population size is used because that size worked well for Lu and Vasko (2015), Zyma et al. (2015) and Vasko et al. (2016) on other combinatorial optimization problems. The first step is to generate 100 permutations of the indices of the problem variables. Next, starting at the beginning of the first permutation of the indices, the corresponding item is inserted into the knapsack as long as all constraints are satisfied. This is continued until all indices have been considered. This results in a feasible solution. For example, if the problem has 250 variables and the first five indices in a permutation of the indices are: $190,15,65,223,99$ then all 250 variables are initially set to zero (the knapsack is empty). We first set $X_{190}=1$, if all constraints are feasible. Next set $X_{15}=1$ and continue until all variables in the permuted list that can be set to one without violating any constraints have been set to one. When constraint(s) violations are encountered, the variable is set back to zero. Then the program moves on to the next variable. In other words, we try to insert each item into the knapsack without violating the constraints based on the permutation order of the variable indices. Once this has been done for the 100 permutations of the indices, the 30 best (highest objective function values) unique feasible solutions compose the initial population of solutions. This is a fast and efficient way to generate an initial population of unique feasible solutions.

\subsection{Binarization Approach}

Keep in mind that TLBO is designed to solve continuous nonlinear optimization problems; whereas, the MKP is a zero-one constrained optimization problem. In other words, the solutions in the population of a TLBO problem will be vectors of real (rational) numbers (usually nonnegative). The solutions in the population for the MKP are bit strings (zeros and ones). To adapt TLBO to deal with bit strings, we used the simple approach that Lu and Vasko (2015) used successfully for the Set Covering Problem. In any of the transformation formulas (teaching or learning), the variables are now bits. The random numbers that took on any values between 0 and 1 now take on only 0 or 1 with equal probability. As in the original TLBO, the teaching factor in TLBO takes on the values 1 or 2 with equal probability. Also, in the teaching phase, the mean solution is replaced by the median solution. Specifically, for our population of 30 solutions, after sorting by objective function, the median solution is taken as solution number 15 (i.e., the $15^{\text {th }}$ best solution out of 30 ). If, after a transformation formula is performed, a variable value is less than 0 , it is set to 0 . If it is greater than 1 , it is set to 1 . Intuitively, if the results of a transformation formula produces a variable that "wants" to have a value less than 0 , we simply set it to 0 . In a like manner, variables that "want" to have a value greater than 1 are set to 1 . The empirical results will demonstrate that this simple binarization approach yields good results. Additionally, it is important to note that there are other (more complicated) approaches in the literature for binarization of metaheuristics originally designed to solve continuous nonlinear optimization problems (Lanza-Gutierrez, 2016). However, Vasko and $\mathrm{Lu}$ (2017) reported that the simple approach outlined above performed the best for the set covering problem.

\subsection{The Repair Operator}

After a transformation formula is used to modify a bit string, this transformed bit string may not represent a feasible solution. We used a variation of the DROP/ADD repair operator commonly used for the MKP (see Chu and Beasley (1998)) to "repair" this bit string, i.e., to make it a feasible solution. If a solution is infeasible because constraints are violated, items are first removed from the knapsack (dropped). Once enough items have been removed so that the solution is now feasible, items are then added as long as the solution remains feasible. Pseudo-utility ratios are defined for each variable based on the following equation: $\mathrm{U}_{\mathrm{j}}=\mathrm{p}_{\mathrm{j}} /\left(\Sigma \mathrm{W}_{\mathrm{i}} \mathrm{a}_{\mathrm{ij}}\right)$ where $\mathrm{W}_{\mathrm{i}}$ are surrogate multipliers or weights. Typically, the $\mathrm{W}_{\mathrm{i}}$ values are obtained from solving the linear programming relaxation of the MKP and using the shadow prices (dual variable values) for constraint $\mathrm{i}$ as the value for $\mathrm{W}_{\mathrm{i}}$. In trying to keep our solution easy to use and 
implement (potentially in a large production system), we chose a simpler way to determine the $\mathrm{W}_{\mathrm{i}}$ values. Specifically, we simply took the best (highest objective function value) solution in the initial population and sorted its constraints by tightness (least slack) in descending order (tightest constraints are at the top of the list). The top half of the constraints have their corresponding $\mathrm{Wi}=1$ and the lower half of the constraints have their corresponding $\mathrm{Wi}=0$. This way of setting the $\mathrm{W}_{\mathrm{i}}$ values means that the user does not have to solve the LP relaxation of the MKP. This might not seem like a big deal, but if a MKP(s) was being solved routinely (e.g., daily) in a larger production system requiring the system to access an LP solver would definitely make the system slower and more complicated. All items are then sorted in descending order based on their $\mathrm{U}_{\mathrm{j}}$ values. The DROP/ADD repair operator consists of two parts. The first part (called DROP) examines each variable starting at the end of the sorted $U_{j}$ list and changes the variable from one to zero if feasibility is violated. The second part (called ADD) reverses the process by examining each variable starting at the beginning of the sorted $\mathrm{U}_{\mathrm{j}}$ list and changes the variable from zero to one as long as feasibility is not violated.

The aim of the DROP part is to obtain a feasible solution from an infeasible solution, while the ADD part tries to improve the objective function value of the feasible solution. Although this approach for determining the $\mathrm{W}_{\mathrm{i}}$ values is simpler (and not as sophisticated) compared to solving the linear programming relaxation of the MKP (especially if the MKP is very large), in solving a number of MKPs using both approaches for determining the $\mathrm{W}_{\mathrm{i}}$ values, the bottom line results were about the same. Hence, our simpler, easier-toimplement approach does not appear to sacrifice performance.

\section{Empirical Results using Beasley's 270 MKPs}

So far in this paper, we have reviewed a "parameter-less" population-based metaheuristics, TLBO (Rao, Savsani and Vakharia (2011)), that was designed to solve continuous nonlinear optimization problems. We then defined a simple procedure for generating an initial population of feasible solutions for the MKP. We then showed how this metaheuristic can be adapted to efficiently solve any binary optimization problems (not just the multidimensional knapsack problem) where the solutions are represented as bit strings. Next, we offered a repair operator for restoring feasibility after solutions are transformed by either phase of TLBO (teaching or learner) that did not require the solution of the linear programming relaxation of the MKP. These steps outline a methodology that can be used by operations research (OR) practitioners (and others) if they need to solve real-world applications requiring the solution of multidimensional knapsack problems. Without a detailed analysis, it is obvious that this methodology is computationally efficient especially for OR practitioners. For example, there is no need for solving the linear relaxation of this problem and no need for any parameter fine-tuning which can be very time consuming. Also, it is important to note, that if a MKP is solved routinely in an industrial production system by an approach that has parameters that need finetuning, the initially tuned parameters will need to be periodically checked in case they need to be adjusted. We have described an efficient, easy to code and implement solution approach for the MKP, but how effective is it on solving large MKPs? To test and compare solution approaches for the MKP, Chu and Beasley (1998) defined 270 MKPs which are available to researchers in Beasley's OR-Library. These 270 problems are divided into 9 datasets with 30 problems in each dataset. In each dataset, there are 10 problems with a tightness ratio of $0.25,10$ problems with tightness ratio of 0.50 and 10 problems with tightness ratio of 0.75. A tightness ratio implies the size of the right hand side value compared to the sum of the variable coefficients for that constraint. For example, a tightness ratio of 0.25 implies that the right hand side of the dimensional constraint is 0.25 times the sum of the variable coefficients for that constraint. The problems consist of either 100, 250 or 500 variables and the number of dimensional constraints are either 5,10 or 30 for a total of nine datasets - one for each combination of the number of variables and number of constraints. Table 1 shows how well our TLBO implementation for the MKP performed on these $270 \mathrm{MKP}$ instances. Specifically, in Table 1 the results are summarized for the 9 data sets. Each entry in the table is the average deviation from optimum over all 30 problems in the dataset. The results for TLBO represent only one execution of this metaheuristic for 300 iterations of the teaching and learning phases. Since there are 30 solutions in the population, the transformation 
formulas were executed $30 \times 2 \times 300 \times$ (the number of variables in the problem) or 18,000 $\mathrm{x}$ (the number of variables in the problem) for each of the 270 MKPs. We used 300 iterations for two reasons; (1) we assumed that if this approach was implemented in a production environment, there would be an execution time limit, (2) preliminary results on the 270 test instances showed negligible improvement by the time 200 iterations were reached. Given that the results in Table 1, can be obtained very quickly (in a few seconds depending on the PC used), and given that the real-world optimal solution will not be known, the OR practitioner could increase his or her confidence in the solution by putting this program in a loop (do not reset the random number seed) and take the best solution. For example, even embedded in a production system, the user can have the program loop say five times (with different initial populations, etc. each time) and have the program return the best overall solution out of the five best solutions obtained by TLBO.

Table 1

Deviation from optimum for TLBO

\begin{tabular}{ccc}
\hline \#CONSTRAINTS & \#VARIABLES & TLBO \\
\hline 5 & 100 & $\mathbf{0 . 4 2}$ \\
5 & 250 & $\mathbf{1 . 4 6}$ \\
5 & 500 & $\mathbf{2 . 5 9}$ \\
10 & 100 & $\mathbf{0 . 6 8}$ \\
10 & 250 & $\mathbf{1 . 4}$ \\
10 & 500 & $\mathbf{1 . 9 7}$ \\
30 & 100 & $\mathbf{0 . 8 3}$ \\
30 & 250 & $\mathbf{1 . 1 3}$ \\
30 & 500 & $\mathbf{1 . 3 3}$ \\
\hline OVERALL AVERAGE & $\mathbf{1 . 3 1}$ \\
\hline
\end{tabular}

Recently, Rezoug et al. (2018) presented a guided genetic algorithm (GGA) to solve the MKP. In their paper, using the 270 MKPs from Beasley's OR-Library, Rezoug et al. (2018) report in their Table 10 how their GGA performed compared to 9 solution approaches from the literature. These results along with our TLBO results are given in Table 2.

Table 2

Deviation from optimum

\begin{tabular}{cccccccccccc}
\hline $\begin{array}{c}\text { \# CONS- } \\
\text { VARS }\end{array}$ & TLBO & GGA & GA & PECH & MAG & VZ & PIR & SCE & CB & NRP & MCF \\
\hline $5-100$ & 0.42 & 0.54 & 1.2 & 4.24 & 8.47 & 7.6 & 1.0 & 2.4 & .59 & .53 & .68 \\
$5-250$ & 1.46 & 0.56 & 1.9 & 4.03 & 5.1 & 4.6 & .31 & 3.03 & .14 & .24 & .26 \\
$5-500$ & 2.59 & 0.54 & 2.1 & 3.83 & 3.37 & 3.0 & .12 & 3.33 & .94 & .08 & .12 \\
$10-100$ & 0.68 & 0.73 & 1.5 & 4.57 & 10.77 & 10.6 & 2.1 & 4.77 & .05 & 1.1 & 1.12 \\
$10-250$ & 1.4 & 0.63 & 1.9 & 3.27 & 7.63 & 6.7 & .67 & 5.03 & .3 & .49 & .48 \\
$10-500$ & 1.97 & 0.57 & 1.9 & 2.9 & 6.03 & 4.97 & .29 & 5.33 & .14 & .19 & .26 \\
$30-100$ & 0.83 & 1.08 & 1.6 & 3.97 & 11.89 & 11.1 & 4.9 & 6.27 & 1.7 & 1.45 & 2.06 \\
$30-250$ & 1.13 & 1.23 & 1.8 & 2.7 & 8.83 & 7.8 & 2.0 & 6.33 & .68 & .8 & .99 \\
$30-500$ & 1.33 & 2.14 & 1.9 & 2.1 & 6.87 & 6.27 & 1.0 & 6.67 & .35 & .49 & .59 \\
\hline OVERALL & 1.31 & 0.89 & 1.8 & 3.51 & 7.66 & 6.95 & 1.4 & 4.8 & .54 & .6 & .73 \\
AVERAGE & & & & & & & & & & & \\
\hline
\end{tabular}

The solution procedures listed in Table 10 of Rezoug et al. (2018) and repeated in our Table 2 are their guided GA, a GA, Primal Effective Capacity Heuristic (PECH), MAG and VZ, two solution approaches using Lagrange multipliers, PIR, a dual surrogate relaxation heuristic with a branch and bound component, Shuffled Complex Evolution (SCE), CB, a GA augmented with a feasibility and constraint 
operator, New Reduction (Pirkul) NRP operates a lagrangian dual relaxation on MKP, and the Modified Choice Function-Late Acceptance Strategy (MCF). The reader should consult Rezoug et al. (2018) for more details. When a paper develops several metaheuristics and empirically evaluates them based on test problem instances available (typically on the WWW) to researchers, usually some statistical analysis is appropriate to determine which results are statistically better than other results. However, metaheuristics are not necessarily compared in a statistical manner to previously published metaheuristics. In this paper, we only developed one metaheuristic. To compare our results with other published metaheuristic results would be very difficult. Either we would have to code these procedures or we would have to request other researchers to share their results or code with us. Both of these seemed restrictive since the purpose of this paper is just to show that our simple TLBO approach for the MKP is reasonably "competitive" with other published MKP solution approaches. In other words, we were not interested in trying to (statistically) prove that our TLBO approach was better than any of the 10 approaches reported in Table 10 of Rezoug et al. (2018), we just wanted to show that it was competitive, while requiring considerably less effort to code, test and implement. From Table 2, we see that our TLBO is competitive with the other 10 solution approaches. We also see from Table 2, that TLBO performs better on the problems with 30 constraints. In Table 3 , we focus on the 30 constraint problem instances (usually an MKP is considered to be more difficult as the number of constraints increase).

Table 3

Deviation from optimum for 30 constraint problem only

\begin{tabular}{cccccccccccc}
\hline $\begin{array}{c}\text { \# CONS- } \\
\text { VARS }\end{array}$ & TLBO & GGA & GA & PECH & MAG & VZ & PIR & SCE & CB & NRP & MCF \\
\hline $30-100$ & 0.83 & 1.08 & 1.6 & 3.97 & 11.89 & 11.1 & 4.9 & 6.27 & 1.7 & 1.45 & 2.06 \\
$30-250$ & 1.13 & 1.23 & 1.8 & 2.7 & 8.83 & 7.8 & 2.0 & 6.33 & .68 & .8 & .99 \\
$30-500$ & 1.33 & 2.14 & 1.9 & 2.1 & 6.87 & 6.27 & 1.0 & 6.67 & .35 & .49 & .59 \\
\hline $\begin{array}{l}\text { OVERALL } \\
\text { AVERAGE }\end{array}$ & 1.1 & 1.5 & 1.8 & 2.9 & 9.2 & 8.4 & 2.6 & 6.4 & .91 & .92 & 1.2 \\
\hline
\end{tabular}

In Table 3, we see that for the larger (usually more difficult) problems, TLBO is actually very competitive. In this case, only two solution procedures have smaller deviations from optimum (and not by much) than TLBO. Furthermore, these two solution procedures that gave slightly better solutions than TLBO for the 90 problem instances with 30 constraints each were definitely more complicated than TLBO. Specifically, the one procedure (CB) used a GA augmented with a feasibility and constraint operator which utilizes problem-specific knowledge and repair operators which locally improve the offspring. The other solution procedure $(\mathrm{NR}(\mathrm{P}))$ that was slightly better than TLBO used a lagrangian dual relaxation combined with a non-trivial dynamic estimation of the core size. TLBO is clearly simpler to code and implement than these two procedures with negligible sacrifice in solution quality.

\section{Summary and Implications for Operations Research Practitioners}

In this paper we discussed an efficient and effective but yet simple to code and implement solution procedure for the multiple dimensional knapsack problem. Specifically, we demonstrated how the "parameter-less" metaheuristic, TLBO (Rao, Savsani and Vakharia (2011)), originally designed to solve continuous nonlinear optimization problems, can be adapted in a straightforward manner to solve binary optimization problems. Furthermore, we demonstrated how this solution procedure can easily be used to effectively solve the multidimensional knapsack problem. Comparing our TLBO solutions for 270 MKPs available from Beasley's OR-Library to published results (Rezoug et al. (2018)) for other MKP solution procedures, we see that TLBO is not only easy to implement and use, but it also gives high quality solutions. TLBO is certainly "competitive" with the other methods discussed in Rezoug et al. (2018). 
A major benefit of our results is that they can easily be used by operations research practitioners to solve industrial problems that cannot be solved efficiently with exact solution procedures. Furthermore, in a real-world situation when the optimal solution is not known, the practitioner can very easily build his/her confidence in the answer obtained from our procedure by simply putting it in a loop and executing it several times (do NOT reset the random number seed) and choosing the best answer obtained.

\section{References}

Akçay, Y., Li, H., \& Xu, S. H. (2007). Greedy algorithm for the general multidimensional knapsack problem. Annals of Operations Research, 150(1), 17.

Baroni, M. D. V., \& Varejão, F. M. (2015, November). A shuffled complex evolution algorithm for the multidimensional knapsack problem. In Iberoamerican Congress on Pattern Recognition (pp. 768775). Springer, Cham.

Boyer, V., Elkihel, M., \& El Baz, D. (2009). Heuristics for the 0-1 multidimensional knapsack problem. European Journal of Operational Research, 199(3), 658-664.

Chu, P. C., \& Beasley, J. E. (1998). A genetic algorithm for the multidimensional knapsack problem. Journal of Heuristics, 4(1), 63-86.

Fréville, A. (2004). The multidimensional 0-1 knapsack problem: An overview. European Journal of Operational Research, 155(1), 1-21.

Frieze, A. M., \& Clarke, M. R. B. (1984). Approximation algorithms for the m-dimensional 0-1 knapsack problem: worst-case and probabilistic analyses. European Journal of Operational Research, 15(1), 100-109.

Baghel, M., Agrawal, S., \& Silakari, S. (2012). Survey of metaheuristic algorithms for combinatorial optimization. International Journal of Computer Applications, 58(19), 2709-2716.

Kellerer, H., Pferschy, U., \& Pisinger, D. (2004). Multidimensional Knapsack Problems. In Knapsack problems(pp. 235-283). Springer, Berlin, Heidelberg.

Kong, X., Gao, L., Ouyang, H., \& Li, S. (2015). Solving large-scale multidimensional knapsack problems with a new binary harmony search algorithm. Computers \& Operations Research, 63, 7-22.

Laabadi, S., Naimi, M., El Amri, H., \& Achchab, B. (2018). The 0/1 Multidimensional Knapsack Problem and Its Variants: A Survey of Practical Models and Heuristic Approaches. American Journal of Operations Research, 8(05), 395.

Labed, S., Gherboudj, A.,\& Chikhi, S. (2011) A Modified Hybrid Particle Swarm Optimization Algorithm for Multidimensional Knapsack Problem, International Journal of Computer Applications, 34(2), 11-16.

Lanza-Gutierrez, J. M., Crawford, B., Soto, R., Berrios, N., Gomez-Pulido, J. A., \& Paredes, F. (2017). Analyzing the effects of binarization techniques when solving the set covering problem through swarm optimization. Expert Systems with Applications, 70, 67-82.

Meng, T., \& Pan, Q. K. (2017). An improved fruit fly optimization algorithm for solving the multidimensional knapsack problem. Applied Soft Computing, 50, 79-93.

Moraga, R. J., DePuy, G. W., \& Whitehouse, G. E. (2005). Meta-RaPS approach for the 0-1 multidimensional knapsack problem. Computers \& Industrial Engineering, 48(1), 83-96.

Newhart, D. D., Stott, K. L., \& Vasko, F. J. (1993). Consolidating product sizes to minimize inventory levels for a multi-stage production and distribution system. Journal of the operational Research Society, 44(7), 637-644.

Rao, R. V., Savsani, V. J., \& Vakharia, D. P. (2011). Teaching-learning-based optimization: a novel method for constrained mechanical design optimization problems. Computer-Aided Design, 43(3), 303-315.

Rezoug, A., \& Boughaci, D. (2016). A self-adaptive harmony search combined with a stochastic local search for the 0-1 multidimensional knapsack problem. International Journal of Bio-Inspired Computation, 8(4), 234-239.

Rezoug A, Bader-El-Den M., \& Boughaci D. (2018) Guided genetic algorithm for the multidimensional knapsack problem, Memetic Computing, 10, 29-42. 
Vasko, F. J., Wolf, F. E., \& Stott, K. L. (1989). A practical solution to a fuzzy two-dimensional cutting stock problem. Fuzzy Sets and Systems, 29(3), 259-275.

Vasko, F. J., Wolf, F. E., \& Pflugrad, J. A. (1991). An efficient heuristic for planning mother plate requirements at Bethlehem Steel. Interfaces, 21(2), 1-7.

Vasko, F. J., Wolf, F. E., Stott, K. L., \& Woodyatt, L. R. (1993). Adapting branch-and-bound for realworld scheduling problems. Journal of the Operational Research Society, 44(5), 483-490.

Vasko, F. J., Newhart, D. D., \& Strauss, A. D. (2005). Coal blending models for optimum cokemaking and blast furnace operation. Journal of the Operational Research Society, 56(3), 235-243.

Vasko, F. J., \& Stott, K. L. (2008). Strategic Planning: OR to the Rescue. OR Insight, 21(3), 26-32.

Vasko, F.J., Lu, Y., \& Zyma, K. (2016). An empirical study of population-based metaheuristics for the multiple-choice multidimensional knapsack problem, International Journal of Metaheuristics, 5(3-4), 193-225.

Vasko, F.J., \& Y. Lu, Y.(2017). Binarization of continuous metaheuristics to solve the set covering problem: Simpler is better. invited talk, 21st Triennial Conference of The International Federation of Operational Research Societies (IFORS), Quebec, Canada, July 17-21, 2017.

Zyma, K., Lu, Y., \& Vasko, F.J. (2015). Teacher training enhances the teaching-learning-based optimization metaheuristic when used to solve multiple-choice multidimensional knapsack problems, International Journal of Metaheuristics, 4(3-4), 268-293.

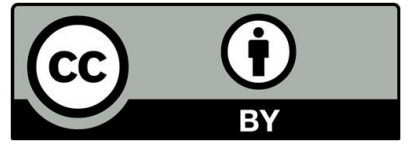

(C) 2019 by the authors; licensee Growing Science, Canada. This is an open access article distributed under the terms and conditions of the Creative Commons Attribution (CCBY) license (http://creativecommons.org/licenses/by/4.0/). 